\title{
Freie Aminosäuren und verwandte Verbindungen in der Rumpfmuskulatur frischgefangener mariner Knochenfische
}

\author{
Aus dem Institut für Meereskunde der Universität Kiel \\ und der Biologischen Anstalt Helgoland \\ Von Heinz Schaefer
}

(Mit 2 Abbildungen und 3 Tabellen)

Ober das Vorkommen von freien Aminosäuren in der Muskulatur von Meeresfischen aus unserem geographischen Bereich ist mehrmals berichtet worden. Die säulenchromatographischen Analysen erfaßten einen großen Teil der Substanzen, jedoch blieben die in nur geringen Konzentrationen vorkommenden Aminosäuren weitgehend unberücksichtigt. Die papierchromatographische Lage von nicht identifizierten Verbindungen wurde nicht beschrieben. SHEWAN analysierte mit dieser Methode Schellfische (1952), weitere Gadiden (1953), Plattfische und Arten aus der Hering-Makrele-Gruppe (1955).

Papierchromatographische Analysen ergaben ein größeres Spektrum von Aminosäuren und verwandten Verbindungen. Jones arbeitete über das Vorkommen von Taurin beim Dorsch (1955) und über die jahreszeitlichen Schwankungen der Konzentrationen einiger freier Aminosäuren im Fleisch des Dorsches (1954) und der Limande (1959). Ranke (1959) beschrieb das Muster der genannten Substanzen in der Muskulatur einer größeren Zahl von Süßwasser-, Brackwasser- und Seefischen aus dem Gebiet von Cuxhaven. Untersuchungen von HugHes (1959) beschäftigten sich mit jahreszeitlichen Unterschieden in den Mengen einer Reihe von Aminosäuren und von Schaefer (1959 und 1961a) mit dem Bestand der freien Aminosäuren vornehmlich bei Rotbarsch, Kabeljau und Schellfisch in Abhängigkeit vom Standort

Die meisten der von den englischen Autoren untersuchten Fische wurden nach dem Fang entweder 6-24 Stunden lang in Seewasseraquarien gehältert oder bis zu 30 Stunden lang in Eis oder bei $0^{0}$ aufbewahrt oder nach Tiefgefrierung analysiert. Die Fische von RANKE gelangten zum größten Teil lebendfrisch zur Bearbeitung.

Der Zweck der vorliegenden Untersuchung ist

1) Erweiterung der Kenntnisse des Grundmusters der freien Aminosäuren in

Deutscher Beitrag zum Polarfrontprogramm des Internationalen Geophysikalischen Jahres 1958.

Der Deutschen Forschungsgemeinschaft danke ich ergebenst für die Gewährung eines Stipendiums und einer Sachbeihilfe.

17 Meeresuntersuchungen Bd. VIII. H. 2 
der Fischmuskulatur durch Untersuchung einer weiteren großen Zahl von Arten aus verschiedenen Meeresgebieten,

2) Untersuchung des Grundmusters direkt nach dem Fang,

3) Versuch einer biologischen Einordnung der Befunde.

Der erste Teil der Untersuchungen, nämlich die Gewinnung der Muskelproben und ihre Konservierung (Aufhebung der Fermenttätigkeit und dadurch Verhinderung von postmortalen Abbau- und Umbauprozessen) war nur an Bord eines Schiffes möglich. Diese Arbeiten konnten an Bord von FFS „Anton Dohrn " während zweier Forschungsfahrten durchgeführt werden.

$\mathrm{Da}$ auch innerhalb der Arten von Fisch zu Fisch schwankende Werte für die einzelnen Substanzen zu erwarten waren, es aber nicht möglich war, von jeder Art eine große Zahl von Fischen zu analysieren, wurde, abgesehen von den quantitativen Stickstoffbestimmungen, die Methode der Papierchromatographie gewählt. Somit ist auch ein Vergleich mit den Ergebnissen einiger anderer Autoren möglich. Die Mengenverhältnisse der einzelnen Substanzen werden dabei unter Verzicht auf die Angabe der absoluten Konzentrationen in mehreren Größenordnungen angegeben, nämlich schwach, mittel, stark und sehr stark, welche sich aus der Größe und der Farbintensität der Flecken auf den Papierchromatogrammen ergeben. Dies genügt, um die einzelnen Arten miteinander vergleichen zu können. Die individuellen Schwankungen der Substanzmengen bei Tieren einer Art aus demselben Fang liegen fast immer innerhalb einer solchen Größenordnung (Schatrer, 1959; in dieser Arbeit werden sogar 5 Größenordnungen angegeben).

Für die Mehrzahl der unbekannten Verbindungen wurde auf eine weitere Analyse verzichtet, da unsere Fragestellung mehr der Artspezifität des Aminosäuremusters galt als der chemischen Struktur der Verbindungen. Jedoch wurden mit diesen Substanzen verschiedene Reaktionen angestellt, um späteren Autoren gewisse Hinweise auf die Natur dieser Stoffe geben zu können, und um Vergleichsgrundlagen für spätere Arbeiten zu bieten.

\section{Methodik}

Wenn nicht anders vermerkt, wurden die Fische direkt nach dem Fang durch Schlag getötet. Durchschnittlich etwa $80 \mathrm{~g}$ (bei kleinen Tieren wurden mehrere zu einer Probe vereinigt) der immer an den gleichen Stellen abpräparierten Rumpfmuskulatur wurden abgewogen, wie üblich unter Zufügung von 96\% \%igem Äthanol im Starmix zerkleinert und unter Zusatz einiger Tropfen Chloroform in Flaschen abgefüllt. Im Landlaboratorium wurde abfiltriert und der Filterrückstand 3-5mal mit heißem 80\%igem Äthanol extrahiert. Die vereinigten Filtrate wurden sodann im Vakuum auf ein kleines Volumen eingeengt. Falls notwendig, erfolgte Entfettung nach Awapara (1948).

Als hauptsächliche Lösungsmittel für die meist zweidimensionale Papierchromatographie, welche vornehmlich mit je zwei Läufen durchgeführt wurde, dienten Butanol-Eisessig-Wasser $(4: 1: 1)$ und Pyridin-Amylalkohol-Wasser (7,5:7:6). Um die Papierchromatogramme der Proben miteinander vergleichen zu können, wurden jeweils Extraktmengen aufgetragen, die $0,1 \mathrm{mg} \mathrm{Kjeldahl-N}$ oder 0,04 mg Amino-N enthielten oder 0,06 g Frischgewicht der Muskulatur entsprachen. Die Angaben in dieser Arbeit beziehen sich vorzugsweise auf die letztgenannte Bezugsgröße. Der Wägefehler beträgt bis etwa $5 \%$. 
Wichtigste Sprühreagentien waren Ninhydrin und Folins Reagens (Natrium- $\beta$-naphthochinonsulfonat). Daneben kamen für Spezialnachweise ein- und zweidimensional zur Verwendung: Reagentien nach Pauly, Ehrlich, Sakagushi und Dragendorff, ferner Isatin, Joddampf sowie Chloramin zum Nachweis von Oxyprolin (Stegemann und Griffin, 1958).

Die Bestimmung des Gesamt-Stickstoffs erfolgte nach KLINGMÜLLER et al. (1955), die des Amino-Stickstoffs nach van Slyke, modif. von Zuwerkalow (1956).

Untersuchte Arten

Ordnung Gadiformes

Kabeljau, Dorsch

Schellfisch

Köhler

Wittling

Blauer Wittling

Seehecht

Lengfisch

Blauleng

Französischer Dorsch

Polardorsch

Brosme, Lumb

Ordnung Anguilliformes

Meeraal

Ordnung Macruriformes

Grenadierfisch

Ordnung Perciformes

Unterordnung Percoidei

Bastardmakrele

Meerbarbe

Seebarsch
Streifenbrasse

Gadus morhua L., Syn.: Gadus callarias L.

Melanogrammus aeglefinus (L.), Syn.: Gadus aegelfinus $\mathrm{L}$.

Pollachius virens (L.), Syn.: Gadus virens L.

Merlangius merlangus (L.), Syn.: Gadus merlangus $\mathrm{L}$.

Micromesistius poutassou (Risso), Syn.: Gadus poutassou Risso

Merluccius merluccius L., Syn.: Merluccius vulgaris Flem.

Molva molva L., Syn.: Molva vulgaris FI.

Molva byrkelange Walbaum

Trisopterus luscus (L.), Syn.: Gadus luscus $-\mathrm{L}$.

Boreogadus saida (Lepechin), Syn.: Gadus saida Lepechin

Brosme brosme Müller, Syn.: Brosmius brosme

Ascanius

Conger conger L., Syn.: Conger vulgaris Cuv.

Macrourus rupestris Gunner

Trachurus trachurus (L.), Syn.: Caranx trachurus L. Spondyliosoma cantharus (L.), Syn.: Cantharus lineatus Mont.

Mullus barbatus surmuletus $\mathrm{L}$.

Roccus (Dicentrarchus) labrax (L.), Syn.: Morone labrax L.

Unterordnung Blennioidei

Katfisch, Steinbeißer

gefleckter Katfisch

Anarhichas lupus L.

Anarhichas minor Olafsen

Lycodes esmarki Collett.

Unterordnung Scombroidei

Makrele
Scomber scombrus L. 
Unterordnung Cottoidei

Knurrhahn

Grauer Knurrhahn

Seehase, Lump
Trigla cuculus L., Syn.: Trigla pini BI

Trigla gurnadus L.

Cyclopterus lumpus L.

Cottunculus microps Coll.

Artediellus spec.

Unterordnung Callionymoidei

Leyerfisch Callionymus lyra L.

Ordnung Clupeiformes

Hering

Glasauge

Clupea harengus $\mathrm{L}$.

Argentina silus Ascanius

Ordnung Lophiiformes

Seeteufel, Angler

Lophius piscatorius $\mathrm{L}$.

Ordnung Heterosomata

Familie Pleuronectidae

Schwarzer Heilbutt Reinhardtius hippoglossoides Walbaum

Doggerscharbe Hippoglossoides platessoides Fabricius, Syn. Drepanopsetta platessoides Walbaum

Limande

Microstomus kitt Walbaum, Syn.: Pleuronectes

microcephalus Donov.

Kliesche

Scholle

Rotzunge

da $\mathrm{L}$

Pleuronectes platessa $\mathrm{L}$.

Glyptocephalus cynoglossus (L.), Syn.: Pleuronectes cynoglossus $\mathrm{L}$.

Familie Bothidae

Steinbutt

Psetta maxima (L.), Syn.: Scophthalmus maximus

L., Rhombus maximus (L.)

Scheefsnut

Familie Soleidae

Seezunge

Lepidorhombus whiff-iagonis Walbaum

Solea solea L., Syn.: Solea vulgaris Quensel

\section{Ergebnisse}

Es wurde das qualitative Vorkommen von freien Aminosäuren und verwandten Verbindungen in der Muskulatur von insgesamt 38 Arten von Knochenfischen untersucht. Quantitative Bestimmungen des Gesamt- und des Aminostickstoffs der alkoholischen Extrakte ergänzten die Befunde. Die Fänge fanden in den Monaten März/April und August/September mit Heringstrawl und Grundschleppnetz in verschiedenen Meeren statt. Die Lage der Fangplätze, angegeben als Stationsnummern laut Fahrtprotokoll des Forschungsschiffes, geht aus den Karten in BöHNECKE und BücKMANN (1959) hervor. Position und hydrographische Angaben sind in Tabelle 1 wiedergegeben.

Der Gesamtstickstoff liegt bei den untersuchten Arten zwischen 70 und $342 \mathrm{mg} / 100 \mathrm{~g}$ Frischgewicht, der Gehalt an Aminostickstoff zwischen 12 und $111 \mathrm{mg} / 100 \mathrm{~g}$ (ein Maximalwert von $256 \mathrm{mg}$ Aminostickstoff wurde bei Gadus saida festgestellt). Eine deutliche Parallelität zwischen Gesamt- und Amino- 


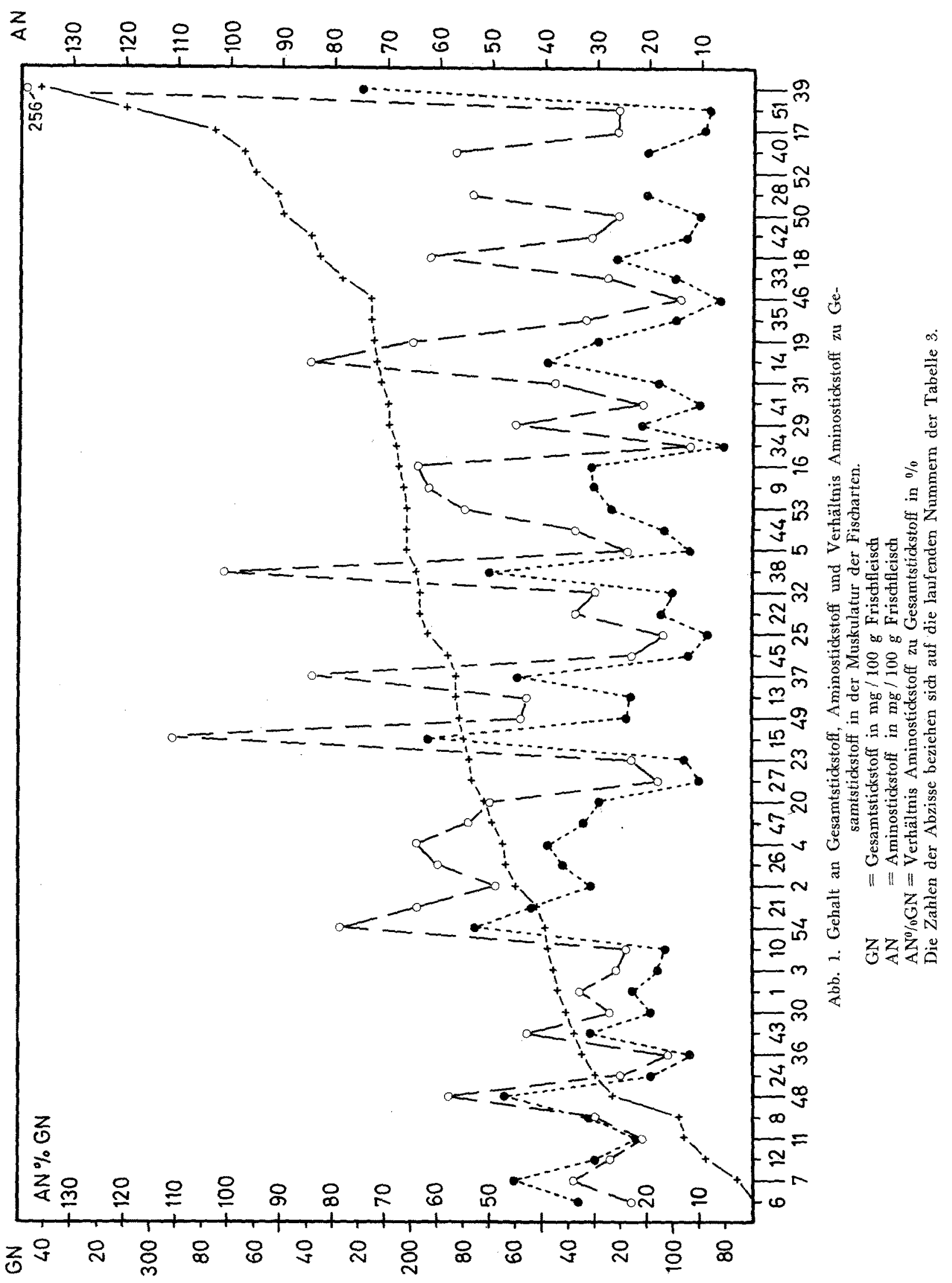


stickstoff besteht nicht (Abb. 1). Hohe Konzentrationen von Gesamtstickstoff fanden sich vornehmlich in der Muskulatur der bei Island-Grönland gefangenen Fische. Die Gesamtstickstoff-Mengen lagen im Sommer meist höher als im Spätwinter. Fische mit geringem Gesamtstickstoff-Gehalt wurden vornehmlich in geringer Wassertiefe gefangen.

Tabelle 1

Hydrographische Angaben zu den Fangstationen

\begin{tabular}{|c|c|c|c|c|c|c|}
\hline Station & Position & & $\begin{array}{c}\text { Salzgehalt } \\
\text { in } \% 0\end{array}$ & $\begin{array}{l}\text { Temperatur } \\
\text { in }^{0} \mathrm{C}\end{array}$ & $\begin{array}{c}\text { Fangtiefe } \\
\text { in } m\end{array}$ & $\begin{array}{l}\text { Wassertiefe } \\
\text { der Messun- } \\
\text { gen in } m\end{array}$ \\
\hline 2232 & $\begin{array}{c}50^{0} 40,5^{\prime} \\
0^{0} 44^{\prime}\end{array}$ & $\begin{array}{l}N \\
0\end{array}$ & 34,83 & 7,25 & $47-50$ & 46 \\
\hline 2233 & $\begin{array}{r}50^{0} 25^{\prime} \\
0^{0} 12^{\prime}\end{array}$ & $\begin{array}{l}\mathrm{N} \\
\mathrm{W}\end{array}$ & 35,07 & 8,48 & $49-56$ & 46 \\
\hline 2234 & $\begin{array}{l}49^{0} 58^{\prime} \\
04^{0} 02^{\prime}\end{array}$ & $\begin{array}{l}\mathrm{N} \\
\mathrm{W}\end{array}$ & 35,10 & 9,62 & 72 & 65 \\
\hline 2264 & $\begin{array}{l}64^{0} 50^{\prime} \\
26^{0} 39^{\prime}\end{array}$ & $\begin{array}{l}\mathrm{N} \\
\mathrm{W}\end{array}$ & 35,08 & $4,34-4,47$ & $220-230$ & $219-235$ \\
\hline 2266 & $\begin{array}{l}65^{0} 36^{\prime} \\
26^{0} 35^{\prime}\end{array}$ & $\begin{array}{l}\mathbf{N} \\
\mathrm{W}\end{array}$ & $35,07-35.08$ & $4,34-5,68$ & $225-235$ & $235-275$ \\
\hline 2274 & $\begin{array}{l}65^{0} 53^{\prime} \\
30^{\circ} 08^{\prime}\end{array}$ & $\begin{array}{l}\mathrm{N} \\
\mathrm{W}\end{array}$ & 34,85 & 1,02 & $400-425$ & 355 \\
\hline 2277 & $\begin{array}{l}65^{0} 38^{\prime} \\
32^{0} 23^{\prime}\end{array}$ & $\begin{array}{l}\mathrm{N} \\
\mathrm{W}\end{array}$ & & & $310-325$ & \\
\hline 2278 & $\begin{array}{l}65^{0} 33^{\prime} \\
32^{0} 15^{\prime}\end{array}$ & $\begin{array}{l}\mathrm{N} \\
\mathrm{W}\end{array}$ & & & $370-400$ & \\
\hline 2301 & $\begin{array}{l}63^{0} 05^{\prime} \\
23^{0} 57^{\prime}\end{array}$ & $\begin{array}{l}\mathrm{N} \\
\mathrm{W}\end{array}$ & 35,19 & 6,45 & $430-460$ & 420 \\
\hline 2305 & $\begin{array}{l}65^{\circ} 00^{\prime} \\
34^{0} 29^{\prime}\end{array}$ & $\begin{array}{l}\mathrm{N} \\
\mathrm{W}\end{array}$ & 35,00 & 4,86 & $420-450$ & 392 \\
\hline 2477 & $\begin{array}{l}54^{0} 58^{\prime} \\
05^{0} 40^{\prime}\end{array}$ & $\begin{array}{l}\mathrm{N} \\
\mathrm{O}\end{array}$ & & & 41 & \\
\hline 2478 & $\begin{array}{r}57^{0} 42^{\prime} \\
0^{0} 12^{\prime}\end{array}$ & $\begin{array}{l}\mathrm{N} \\
\mathrm{W}\end{array}$ & & & 95 & \\
\hline 2479 & $\begin{array}{l}57^{0} 26^{\prime} \\
13032^{\prime}\end{array}$ & $\begin{array}{l}\mathrm{N} \\
\mathrm{W}\end{array}$ & 35,37 & 9,04 & 156 & 145 \\
\hline 2523 & $\begin{array}{l}65^{0} 59^{\prime} \\
28^{0} 32^{\prime}\end{array}$ & $\begin{array}{l}\mathrm{N} \\
\mathrm{W}\end{array}$ & 34,89 & 0,11 & 380 & 395 \\
\hline 2526 & $\begin{array}{l}66^{0} 40^{\prime} \\
30^{0} 14^{\prime}\end{array}$ & $\begin{array}{l}\mathbf{N} \\
\mathrm{W}\end{array}$ & 34,88 & 0,40 & 400 & 410 \\
\hline 2536 & $\begin{array}{l}65^{0} 32,5^{\prime} \\
32^{0} 12^{\prime}\end{array}$ & $\begin{array}{l}\mathrm{N} \\
\mathrm{W}\end{array}$ & 35,07 & 5,87 & 400 & 391 \\
\hline 2557 & $\begin{array}{l}63^{0} 16,5^{\prime} \\
22^{0} 15^{\prime}\end{array}$ & $\begin{array}{l}\mathrm{N} \\
\mathrm{W}\end{array}$ & & 7,26 & 300 & 290 \\
\hline 2558 & $\begin{array}{l}63^{0} 04^{\prime} \\
23^{0} 59^{\prime}\end{array}$ & $\begin{array}{l}N \\
W\end{array}$ & & 7,28 & 450 & 450 \\
\hline 2568 & $\begin{array}{l}65^{\circ} 09^{\prime} \\
33^{0} 51^{\prime}\end{array}$ & $\begin{array}{l}\mathrm{N} \\
\mathrm{W}\end{array}$ & & & 450 & \\
\hline 2598 & $\begin{array}{l}62^{0} 30^{\prime} \\
40^{\circ} 30^{\prime}\end{array}$ & $\begin{array}{l}\mathrm{N} \\
\mathrm{W}\end{array}$ & & & $240-270$ & \\
\hline 2620 & $\begin{array}{l}59^{0} 41^{\prime} \\
44^{0} 12^{\prime}\end{array}$ & $\begin{array}{l}\mathrm{N} \\
\mathrm{W}\end{array}$ & $33,73-33,51$ & $2,65-3,65$ & $125-135$ & $120-125$ \\
\hline 2649 & $\begin{array}{l}57^{0} 42^{\prime} \\
09^{09} 3^{\prime}\end{array}$ & $\begin{array}{l}\mathrm{N} \\
\mathrm{W}\end{array}$ & & 10,24 & 207 & 168 \\
\hline
\end{tabular}

Soweit gleichzeitig hydrographische Messungen gemacht wurden, besorgte mir Herr Prof. K. Kalle, Hamburg, diese Angaben. In einigen Fällen wurden mangels Angaben die Meßergebnisse von sehr benachbarten Stationen mit entsprechender Tiefe eingetragen. 
Bei dem Aminostickstoff sind diese Beziehungen nicht ausgeprägt. Es ist hier lediglich auffällig, daß alle als "arktisch" einzuordnenden Arten - allerdings neben mediterranen - die höchsten Konzentrationen aufwiesen. Hierbei ist beachtenswert, daß alle Arten einschließlich Rotbarsch, die auf Station 2523 gefangen wurden, sehr hohe Aminostickstoff-Gehalte besaßen. Hier wurde die niedrigste Wassertemperatur gemessen. Auf einer etwas weiter nordwest-

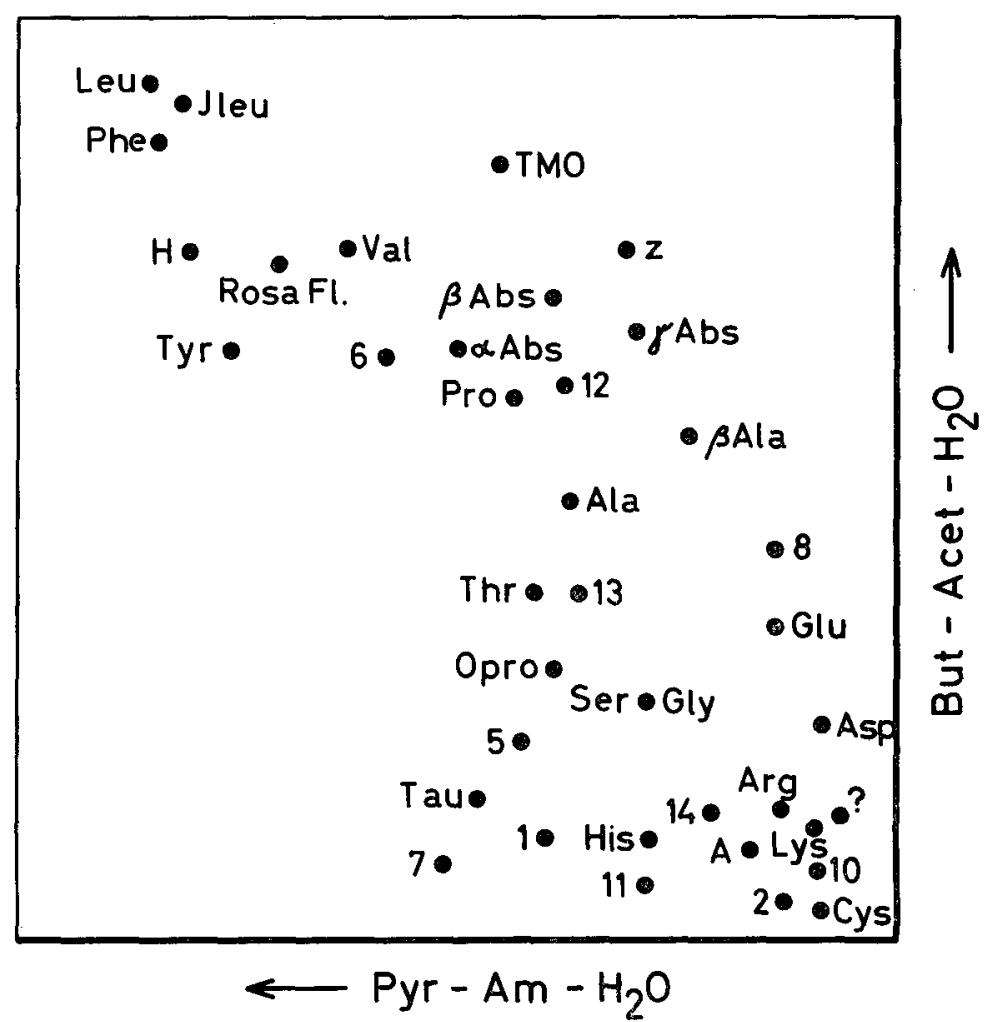

Abb. 2. Schematisches Papierchromatogramm mit Angabe der Positionen der nicht-indentifizierten Substanzen.

$\mathrm{A}=$ Anserin, $\mathrm{H}=$ Harnstoff. Die übrigen Abkürzungen siehe Tabelle 3.

Lösungsmittel: Butanol-Eisessig-Wasser $4: 1: 1$

Pyridin-Amylalkohol-Wasser $7,5: 7: 6$

wärts gelegenen Station (2526) mit ebenfalls niedriger Temperatur lagen die Aminostickstoff-Werte wesentlich tiefer. Die Schwankungen der Menge des Gesamt- und Aminostickstoffs waren bei Fischen gleicher Art, aber von verschiedenen Fangplätzen, stärker als bei solchen aus ein und demselben Fang. Eine Beziehung zwischen der Menge des Gesamtstickstoffs einerseits und der Quantität der einzelnen Aminosäuren sowie der Wassertemperatur andererseits ist nicht erkennbar. Hingegen sind die papierchromatographisch geschätzten Mengen von Glycin, Glutaminsäure, Asparaginsäure, Taurin, Alanin und Threonin um so höher, je mehr Aminostickstoff vorhanden ist. Für die übrigen Aminosäuren trifft dies nicht zu. Zwischen Aminostickstoff und der Wassertemperatur, Fangtiefe und der Aktivität können keine Beziehungen erkannt werden. 
Die höchsten Anteile des Aminostickstoffs am Gesamtstickstoff wiesen arktische Fische auf.

Distinkte Unterschiede zwischen Männchen und Weibchen sind weder durch die quantitativen Stickstoff-Bestimmungen noch papierchromatographisch erkennbar. In einigen Fällen waren zwar Differenzen in der Größe der Flecken von Prolin und Leucin festzustellen, doch reicht die Zahl der untersuchten Tiere nicht zu einer klaren Beweisführung aus.

Auf den Papierchromatogrammen wurden neben den bekannten Aminosäuren weitere Substanzen festgestellt, die vermutlich seltenere Aminosäuren, Peptide, Amine oder andere verwandte Verbindungen darstellen. Zum Teil sind sie nicht ninhydrinpositiv, sondern wurden mit anderen Farbreaktionen nachgewiesen. Sie werden mit Ziffern oder Buchstaben bezeichnet. Ihre papierchromatographische Lage zeigt die schematische Abbildung 2. Einige von ihnen, nämlich „PR“, „g.b.F.“, „1“, „2“, „3“, „4“, „5“, „,““, „Rosa Fleck“ sind schon von RANKE (1959) beschrieben worden. „PR“ wurde inzwischen von Suryanarayaná Rao (1960) als Trimethylaminoxyd erkannt. Nach Ranke

Tabelle 2

Reaktionen von unbekannten Substanzen

\begin{tabular}{|c|c|c|c|c|c|c|c|c|}
\hline 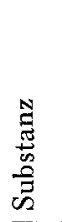 & 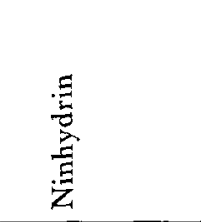 & $\stackrel{\Xi}{\circ}$ & $\frac{\bar{E}}{\stackrel{5}{c}}$ & 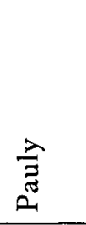 & 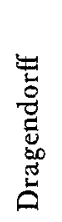 & 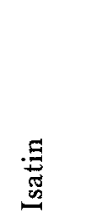 & 范 & 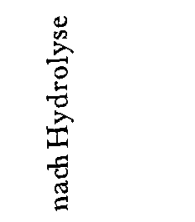 \\
\hline 1 & $\begin{array}{l}\text { gelbbraun- } \\
\text { purpur }\end{array}$ & rot & & rot & rot & & & unverändert \\
\hline 2 & purpur & gelbbraun & & & & & & unverändert. \\
\hline 5 & $\begin{array}{l}\text { purpur, etwas } \\
\text { rötlicher als } \\
\text { Taurin }\end{array}$ & $\begin{array}{l}\text { etwas blauer } \\
\text { als Taurin }\end{array}$ & & & & & & unverändert \\
\hline 6 & purpur & blau & & & rot & & & verschwunden \\
\hline 10 & purpur & grünblau & & & & & & unverändert \\
\hline 11 & purpur & $\begin{array}{l}\text { orange- } \\
\text { braunrot }\end{array}$ & gelb & & & blau & & unverändert \\
\hline 13 & rötlich & gelborange & & weiß & & & karmin & unverändert \\
\hline 14 & purpur & rotgelb & & & rot & & rot & unverändert \\
\hline$z$ & purpur & hellblau & & gelbg: & ün & & & verschwunden \\
\hline
\end{tabular}

Fehlende Angaben bedeuten negative oder nicht eindeutige Befunde.

verschwinden „g.b.F.“, „Rosa Fleck“ und „z“ nach saurer Hydrolyse. Zum $Z$ weck einer weiteren Identifizierung wurden die wichtigsten der unbekannten Flecken mit verschiedenen Reagentien behandelt und der Hydrolyse unterworfen. Die Ergebnisse zeigt Tabelle 2. Ob und welche dieser unbekannten Flecke mit nicht identifizierten Substanzen von SHEwaN $(1953,1955)$ übereinstimmen, kann wegen unterschiedlicher Methoden nicht festgestellt werden. Einige der unbekannten Verbindungen sind nur bei je zweimaligem Lauf in den benutzten Lösungsmitteln nachweisbar.

Fleck „?" ergibt bei Behandlung mit Folins Reagens eine von dem be- 
nachbartem Lysin abweichende Färbung. Mischchromatographie mit Ornithin ergibt identischen Fleck. Es dürfte sich bei „?" also um Ornithin handeln.

„g.b.F." wurde ebenfalls weiter untersucht. 1) Eine Probe der Doggerscharbe wurde je 14 Tage lang wiederholt in Butanol-Eisessig-Wasser und in Pyridin-Amylalkohol-Wasser zweidimensional entwickelt. Die betreffenden Flecken wurden aus einer Reihe von Chromatogrammen ausgeschnitten, eluiert und mit $6 \mathrm{n}$ Salzsäure hydrolysiert. Hydrolysenprodukte waren B-Alanin und Methylhistidin. 2) Die aus den Extrakten von Köhler und Knurrhahn gewonnene Substanz verließ eine Dowex-X-50 - Säule nach HamiltonANDERSEN (1959) zwischen Lysin und Histidin. Nach Hydrolyse wurden ß-Alanin und Methylhistidin festgestellt. 3) Diese Untersuchungen wurden bestätigt durch Papierchromatographie der hydrolysierten Extrakte weiterer Fischarten. Der Methylhistidinfleck war immer Pauly-negativ. Die Identität von "g.b.F." mit dem Peptid Anserin wird dadurch wahrscheinlich gemacht. Dicht neben dem bei allen Arten gefundenen "Rosa Fleck" fand sich ein Fleck, der mit Ehrlichs Reagens anfärbbar war. Die Substanz erwies sich als identisch mit Harnstoff.

Die Substanz „5“ ist von Taurin mit den hier benutzten Lösungsmittelgemischen wegen benachbarten Rf-Wertes nicht immer leicht unterscheidbar. Daß „5“ mit Taurin nicht identisch ist, ergibt sich ebenfalls aus etwas abweichender Anfärbbarkeit mit Folins Reagens sowie durch weitere Trennung mit anderen Lösungsmitteln.

Vergleicht man die Ergebnisse der papierchromatographischen Analyse der verschiedenen Arten miteinander, dann zeigt sich hinsichtlich des Vorkommens der bekannteren Aminosäuren qualitativ eine ziemliche Übereinstimmung aller Arten (Tabelle 3). Allerdings konnten einige Aminosäuren nicht oder nur zuweilen nachgewiesen werden, da sie entweder gar nicht oder in nur schwachen Konzentrationen vorhanden waren. Qualitative Unterschiede zeigen sich im Bestand der noch nicht identifizierten Verbindungen. Es ist jedoch deutlich ersichtlich, daß die Mengenverhältnisse der verschiedenen freien Aminosäuren, ausgedrückt durch Fleckengröße und Farbintensität, sehr stark von Art zu Art variieren. Die erheblichsten Mengen von freien Aminosäuren waren bei den aktiven Fischen Makrele, Hering, Bastardmakrele sowie bei einigen trägen Arten wie Wolfsfisch und Katfisch, die geringsten beim Glasauge zu erkennen.

In nur schwachen Mengen oder überhaupt nicht wurden gefunden: Phenylalanin, Methionin, Tryptophan, Tyrosin und - häufig - die Aminobuttersäuren. In mittleren oder schwachen Konzentrationen kamen durchschnittlich vor Asparaginsäure und Arginin, mittel bis stark Lysin, Cystin, Threonin und Valin, stark Alanin, Taurin, Glycin und Glutaminsäure. Von Art zu Art sehr stark schwankend waren die Mengen von Prolin, Oxyprolin, Leucin/Isoleucin, ß-Alanin und Histidin. Eine saubere Trennung von Glycin und Serin wurde nur in wenigen Fällen erzielt, diesbezügliche Angaben betreffen also den Glycin-Serin-Komplex. Nicht immer erfolgreich war ferner die Trennung von Leucin-Isoleucin, so daß es in diesen Fällen dahingestellt bleiben muß, ob es sich um Leucin oder Isoleucin oder um beide Aminosäuren handelt.

Im folgenden sollen einige auffälligere Befunde mitgeteilt werden. Die größten Mengen von Histidin traten bei den aktivsten Fischen sowie bei einigen anderen Arten auf. Alle Fische mit starkem Histidin-Vorkommen wurden 


\begin{tabular}{|c|c|c|c|c|c|c|c|c|c|c|c|c|}
\hline \multicolumn{2}{|c|}{$\begin{array}{l}\text { Lfd. Art } \\
\text { Nr. }\end{array}$} & Station & Datum & $\begin{array}{c}\mathrm{mg} \mathrm{GN} \mathrm{in} \\
100 \mathrm{~g} \mathrm{~F}\end{array}$ & $\begin{array}{c}\mathrm{mg} \mathrm{AN} \text { in } \\
100 \mathrm{~g} \mathrm{~F}\end{array}$ & $\mathrm{AN} \% \mathrm{GN}$ & $\begin{array}{c}\text { Rest-N } \\
\text { mg }\end{array}$ & Ser-Gly & Lys & Cys & Glu & Asp \\
\hline \multicolumn{13}{|c|}{ Kanal } \\
\hline 1 & Lengfisch & 2232 & 1.3. & 144 & 38 & 22,9 & 111 & $+4+$ & +1 & $+t+$ & $+1+$ & $t+$ \\
\hline 2 & Wittling & 2232 & 1. 3. & 160 & 49 & 30,7 & 111 & $+t+$ & +++ & +++ & +++ & ++ \\
\hline 3 & Franz. Dorsch & 2232 & 1.3. & 146 & 26 & 17,9 & 120 & $+\frac{1}{4}+$ & + & + & $+t+$ & $t+$ \\
\hline 4 & Leyerfisch & 2232 & 1.3. & 165 & 64 & 39,0 & 101 & $+1+$ & +1 & +1 & $++\frac{3}{1}$ & $t+t$ \\
\hline 5 & Seczunge & 2232 & 1.3. & 202 & 24 & 12,0 & 178 & $++t$ & $+1+$ & +++ & $+t+$ & ++ \\
\hline 6 & Knurrhahn & 2233 & 1.3 & 70 & 23 & 33,1 & 47 & ++ & $+t+$ & + & ++ & ++ \\
\hline 7 & Scholle & 2233 & 1.3. & 76 & 34 & 45,3 & 42 & $+1+$ & +4 & $+1+$ & $+t+$ & ++ \\
\hline 8 & Seebarsch & 2233 & 1.3. & 98 & 30 & 31,0 & 68 & $+1+$ & +++ & + & +++ & $+1+$ \\
\hline 9 & Meeraal & 2233 & 1.3. & 203 & 62 & 30,4 & 141 & $+t+$ & $+t+$ & + & $+\frac{1}{t}+$ & ++ \\
\hline 10 & Meerbarbe & 2233 & 1.3. & 148 & 24 & 16,6 & 124 & $+1+$ & $+4+$ & + & ++4 & +1 \\
\hline 11 & Bastardmakrele & 2234 & 2.3. & 96 & 21 & 22,0 & 75 & $+t+$ & $+t+$ & ++ & $++t$ & + \\
\hline 12 & Streifenbrasse & 2234 & 2.3 & 88 & 27 & 30,3 & 61 & $+t+$ & + & $+t+$ & $++t$ & $t+$ \\
\hline \multicolumn{13}{|c|}{ Nordsee } \\
\hline 13 & Steinbutt & 2477 & 6.8. & 183 & 43 & 23,4 & 140 & +++ & ++ & $+t+$ & $+t+$ & + \\
\hline 14 & Grauer Knurrhahn & 2477 & 6.8 & 214 & 84 & 39,4 & 130 & $+1+$ & + & $+t$ & +++ & + \\
\hline 15 & Makrele & 2478 & 7. 8. & 180 & 111 & 61,7 & 69 & $+t+$ & $+t+$ & ++ & $++t$ & +++ \\
\hline 16 & Hering & 2478 & 7.8. & 205 & 64 & 31,2 & 141 & +++ & +++ & +++ & $t+t$ & + \\
\hline 17 & Rotzunge & 2478 & 7.8 & 276 & 26 & 9,4 & 250 & $+t+$ & + & + & $+t$ & + \\
\hline 18 & Doggerscharbe & 2478 & 7.8. & 286 & 62 & 26,2 & 174 & ++1 & +++ & +++ & +++ & ++ \\
\hline 19 & Kliesche & 2478 & 7.8. & 215 & 64 & 29,7 & 151 & +++ & ++ & +1 & $+t+$ & ++ \\
\hline 20 & Seeteufel & 2478 & 7.8 & 172 & 50 & 29,2 & 122 & $+t+$ & $+t+$ & $+t+$ & $+t+$ & +1 \\
\hline 21 & Bastardmakrele & 2478 & 7.8. & 152 & 64 & 42,1 & 88 & $+4+$ & +++ & +++ & $+t+$ & $++t$ \\
\hline \multicolumn{13}{|c|}{ Westlich Schottland } \\
\hline 22 & Schellfisch & 2479 & 9.8 & 197 & 34 & 17,5 & 163 & $+1+$ & $+t$ & ++ & $+t$ & + \\
\hline 23 & Scheefsnut & 2479 & 9.8 & 178 & 23 & 12,9 & 155 & +++ & + & ++ & ++ & + \\
\hline 24 & Grauer Knurrhahn & 2479 & 9.8 & 130 & 25 & 19,3 & 105 & $t+t$ & + & $+t$ & $t+$ & $+t$ \\
\hline 25 & Scheef snut & 2649 & 23.9 & 194 & 17 & 8,7 & 173 & $+4+$ & + & +4 & ++ & + \\
\hline 26 & Köhler & 2649 & 23.9. & 164 & 60 & 36,2 & 104 & +++ & ++ & ++ & $++t$ & + \\
\hline 27 & Seehecht & 2649 & 23.9 & 177 & 18 & 10,2 & 159 & $t+t$ & $+t$ & ++ & ++ & + \\
\hline 28 & Limande & 2649 & 23.9 & 252 & 54 & 20,6 & 198 & $+t+$ & $+4+$ & $+4+$ & $+1+$ & $\frac{1}{t}+$ \\
\hline \multicolumn{13}{|c|}{ Island-Grönland } \\
\hline 29 & Kabeljau & 2264 & 16.3. & 209 & 45 & 21,3 & 164 & $+4+$ & + & + & $+t+$ & + \\
\hline 30 & Katfisch, gefl. & 2266 & 16.3. & 141 & 27 & 19,4 & 114 & +++ & $+t+$ & +++ & +++ & $++t$ \\
\hline 31 & Blauer Wittling & 2274 & 18.3. & 212 & 38 & 18,0 & 174 & +++ & +++ & +++ & +++ & + \\
\hline 32 & Kabeljau & 2277 & 19.3 & 197 & 30 & 15,4 & 167 & $+t+$ & +4 & ++ & $+4+$ & + \\
\hline 33 & Grenadierfisch & 2278 & 19.3. & 227 & 28 & 12,5 & 199 & $+t+$ & $+t$ & $+t+$ & $++t$ & ++ \\
\hline 34 & Glasauge & 2301 & 28.3 & 206 & 12 & 6,0 & 194 & ++ & + & ++ & +++ & + \\
\hline 35 & Blauleng & 2301 & 28.8 & 216 & 32 & 14,7 & 184 & $+t+$ & $+t$ & +4 & $+1+$ & + \\
\hline 36 & Brosme & 2305 & 4.4. & 135 & 16 & 11,8 & 119 & ++1 & +++ & +++ & $+t+$ & + \\
\hline 37 & Wolfsfisch & 2523 & 21.8. & 183 & 84 & 45,0 & 99 & +++ & +++ & +++ & +++ & ++ \\
\hline 38 & Cottunculus & 2523 & 21.8. & 198 & 101 & 50,4 & 97 & $-t+$ & $+t$ & $+t+$ & $+t+$ & $+t+$ \\
\hline 39 & Polardorsch & 2523 & 21.8. & 343 & 256 & 74,7 & 87 & $+4+$ & + & +++ & +++ & $+t+$ \\
\hline 40 & Artediellus & 2523 & 21.8. & 265 & 57 & 20,4 & 208 & +++ & $+t+$ & +++ & $++t$ & + \\
\hline 41 & Schwarzer Heilbutt & 2526 & 22.8 & 209 & 21 & 10,3 & 188 & $+1+$ & $+t+$ & $+t+$ & ++1 & +1 \\
\hline 42 & Doggerscharbe & 2526 & 22.8. & 239 & 31 & 12,8 & 208 & $+t+$ & ++ & +++ & $+t+$ & + \\
\hline 43 & Katfisch & 2536 & 23.8 & 138 & 43 & 31,0 & 95 & +++ & $+t+$ & +++ & $++t$ & + \\
\hline 44 & Brosme & 2536 & 23.8 & 202 & 34 & 16,9 & 168 & $+t+$ & $+t+$ & $+1+$ & $+t+$ & + \\
\hline 45 & Blauleng & 2557 & 29.8 & 186 & 23 & 12,5 & 163 & $+t+$ & $+\frac{1}{1}$ & ++ & ++4 & + \\
\hline 46 & Rotzunge & 2557 & 29.8. & 216 & 14 & 6,6 & 102 & $+t+$ & ++ & ++ & ++ & + \\
\hline 47 & Brosme & 2558 & 29.8 & 169 & 54 & 32,2 & 115 & $++t$ & ++ & ++ & $+t+$ & + \\
\hline 48 & Blauleng & 2558 & 29.8 & 123 & 58 & 47,3 & 71 & $+t+$ & $t+t$ & $+1+$ & $+t+$ & $t+$ \\
\hline 49 & Katfisch & 2568 & 4. 9 & 182 & 44 & 24,0 & 138 & $++t$ & +++ & +++ & +++ & ++ \\
\hline 50 & Blauer Wittling & 2568 & 4.9 & 250 & 26 & 10,5 & 224 & $+t+$ & $+t+$ & $+t+$ & $+t$ & + \\
\hline 51 & Kabeljau & 2598 & 10.9 & 310 & 26 & 8,4 & 284 & $+4+$ & $+t$ & + & $+t+$ & + \\
\hline 52 & Kabeljau & 2620 & 15.9. & 261 & & & & ++1 & ++ & ++ & ++ & + \\
\hline 53 & Doggerscharbe & 2620 & 15.9. & 202 & 55 & 27,3 & 147 & $+t+$ & +++ & +++ & $+\frac{1}{t}+$ & ++ \\
\hline 54 & Seehase & 2620 & 15,9 . & 149 & 79 & 52,8 & 70 & $+4+$ & $+t+$ & $+1+$ & $+t+$ & $+t$ \\
\hline
\end{tabular}

Abkürzungen: $\quad$ GN in $100 \mathrm{~g} \mathrm{~F}=$ Gesamtstickstoff der Extrakte in $100 \mathrm{~g}$ Frischgewicht AN in $100 \mathrm{~g} \mathrm{~F}=$ Aminostickstoff der Extrakte in $100 \mathrm{~g}$ Frischgewicht Ser-Gly $=$ Serin-Glycin, Lys $=$ Lysin, Cys $=$ Cystein $/$ Cystin Glu $=$ Glutaminsäure, Asp $=$ Asparaginsäure, His = Histidin,
Arg $=$ Arginin, Tau $=$ Taurin Opro = Oxyprolin, Thr $=\mathrm{Th}$ $\mathrm{Abs}=$ Aminobuttersäure, Leu TMO = Trimethylaminoxyd, 
Tabelle 3

Analysenergebnisse der frischgefangenen Fische

His Arg Tau Ala B-Ala Pro Opro Thr Tyr $\quad$ Val $\alpha$-Abs $\quad \beta$-Abs $\quad \gamma$-Abs $\quad$ Leu Ileu

\begin{tabular}{|c|c|c|c|c|c|c|c|c|c|}
\hline+ & + & +++ & +++ & & $\div$ & + & ++ & + & +++ \\
\hline++ & + & $++t$ & +++ & ++ & ++ & + & $+t+$ & & +++ \\
\hline t. & + & $t+t$ & $t+$ & $t+$ & + & + & + & + & ++ \\
\hline++ & + & +++ & $+t+$ & + & + & + & $t+$ & + & ++ \\
\hline+++ & $+t+$ & +++ & +++ & + & ++ & + & +++ & & $+t+$ \\
\hline+ & ++ & +++ & ++ & + & + & + & + & & + \\
\hline+ & + & $+十+$ & +++ & + & + & + & $t+t$ & & ++ \\
\hline+++ & + & $++t$ & $+t+$ & ++ & ++ & + & +++ & + & + \\
\hline$+\cdot+$ & ++ & +++ & $++t$ & +++ & +++ & ++ & +++ & + & $t+$ \\
\hline$+1+$ & ++ & $t+t$ & $++t$ & & ++ & + & ++ & & ++ \\
\hline+++ & + & $++t$ & $++t$ & + & + & + & ++ & + & ++ \\
\hline$+t+$ & ++ & $++t$ & +++ & + & ++ & + & ++ & + & \\
\hline
\end{tabular}

\begin{tabular}{|c|c|c|}
\hline+ & ++ & $+t$ \\
\hline+ & \multicolumn{2}{|c|}{++} \\
\hline+ & $+t$ & $+t$ \\
\hline+ & \multicolumn{2}{|c|}{+} \\
\hline+ & ++ & ++ \\
\hline+ & \multicolumn{2}{|c|}{+} \\
\hline+ & \multicolumn{2}{|c|}{++} \\
\hline+ & + & + \\
\hline+ & ++ & + \\
\hline+ & ++ & ++ \\
\hline+ & ++ & ++ \\
\hline+ & ++ & ++ \\
\hline
\end{tabular}

$\begin{array}{lllll}+ & + & +++ & +++ & + \\ +++ & ++ & +++ & +++ & + \\ ++++ & & +++ & +++ & ++ \\ ++++ & + & +++ & +++ & ++ \\ + & + & +++ & +++ & + \\ +++ & + & +++ & +++ & + \\ ++ & + & +++ & +++ & ++ \\ + & + & +++ & +++ & ++ \\ ++t+ & & +++ & +++ & +\end{array}$

$\begin{array}{llllll}+ & + & ++ & + & ++ & + \\ +++ & ++ & ++ & & ++ & + \\ ++ & + & +++ & + & +++ & + \\ ++ & + & +++ & & +++ & + \\ ++ & + & ++ & & ++ & + \\ ++ & +++ & ++ & + & ++ & + \\ + & ++ & +++ & + & ++ & + \\ +++ & + & +++ & & ++ & + \\ ++ & + & +++ & + & +++ & +\end{array}$
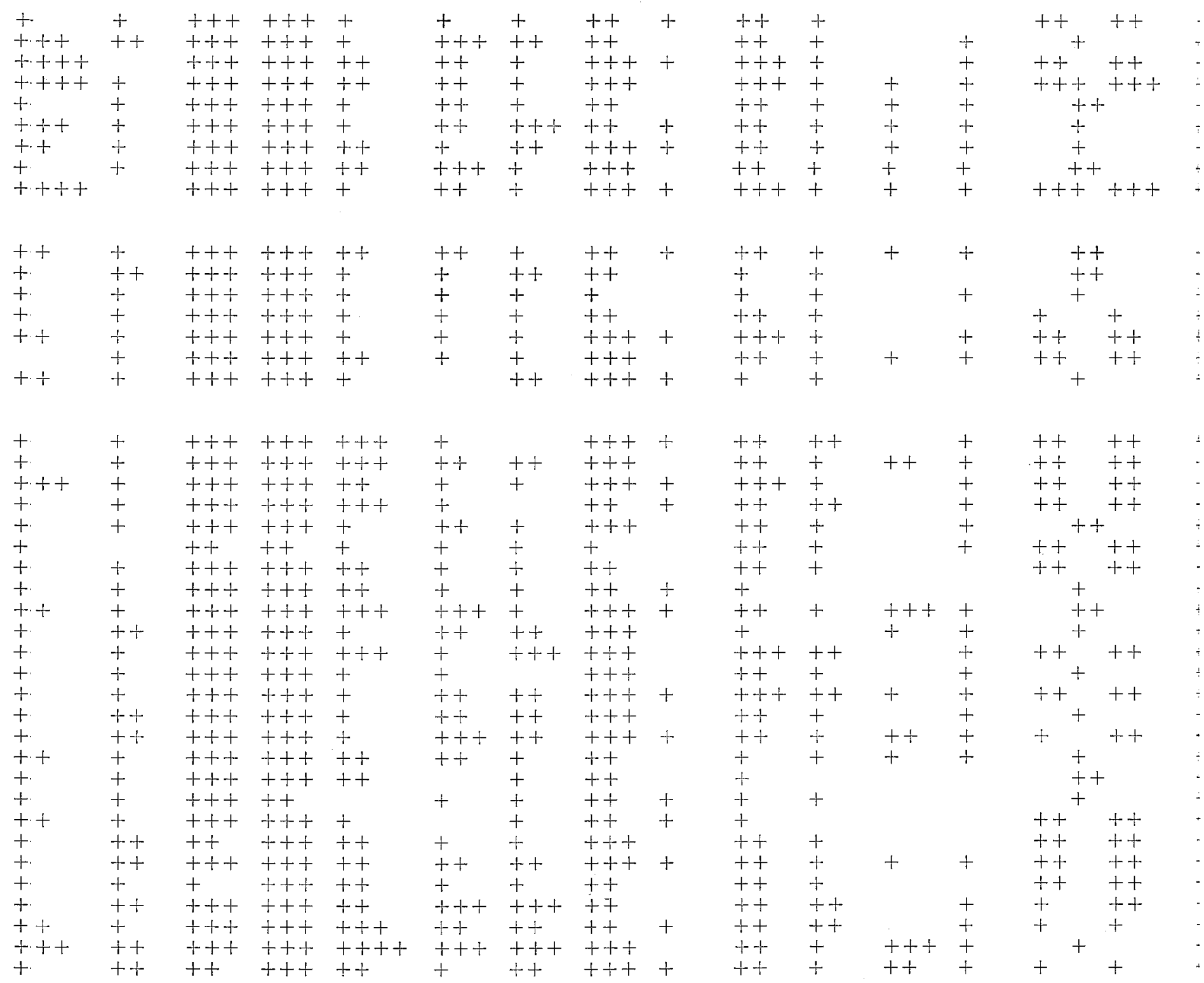

, Ala $=$ Alanin, Pro $=$ Prolin, feonin, $\mathrm{Tyr}=$ Tyrosin, Val $=$. Valin, 


\begin{tabular}{|c|c|c|c|c|c|c|c|c|c|c|c|c|c|c|}
\hline TIMO & Anserin & 1 & 2 & 5 & 6 & 7 & 8 & 10 & 11 & 12 & 13 & 14 & $z$ & $?$ \\
\hline $\begin{array}{l}++ \\
+++ \\
+++ \\
++ \\
+++ \\
+++ \\
+ \\
+++ \\
+++ \\
+++ \\
+++ \\
++\end{array}$ & $\begin{array}{l}+ \\
+++ \\
+++ \\
+ \\
++ \\
+++\end{array}$ & $\begin{array}{l}++ \\
+ \\
+ \\
++ \\
++ \\
+ \\
++ \\
++ \\
+++ \\
+++ \\
+\end{array}$ & $\begin{array}{l}+ \\
+++\end{array}$ & $\begin{array}{l}+++ \\
+++ \\
+++ \\
+++ \\
+++ \\
+++ \\
+++ \\
+++ \\
+++ \\
++ \\
+++ \\
+++\end{array}$ & $\begin{array}{l}+ \\
+ \\
++ \\
+ \\
+\end{array}$ & ++ & $\begin{array}{l}+ \\
+ \\
+\end{array}$ & ++ & $\begin{array}{l}+ \\
+ \\
+\end{array}$ & ++ & & $+t$ & $\begin{array}{l}+ \\
+ \\
+\end{array}$ & ++ \\
\hline $\begin{array}{l}+++ \\
++ \\
+++ \\
+++ \\
+++ \\
++ \\
+++ \\
+++ \\
+++\end{array}$ & ++ & $\begin{array}{l}+ \\
+++ \\
+++ \\
+ \\
+ \\
+++ \\
+++ \\
+ \\
+++\end{array}$ & $\begin{array}{l}+ \\
+\end{array}$ & $\begin{array}{l}+++ \\
+++ \\
+++ \\
+++ \\
+++ \\
+++ \\
+++ \\
+++ \\
+++\end{array}$ & $\begin{array}{l}+ \\
+ \\
+ \\
+ \\
+ \\
+ \\
+ \\
+ \\
+\end{array}$ & ++ & $\begin{array}{l}+ \\
+\end{array}$ & $\begin{array}{l}+++ \\
+ \\
+++ \\
+ \\
+++\end{array}$ & $\begin{array}{r}+ \\
+\end{array}$ & & ++ & & $\begin{array}{l}+ \\
+ \\
+\end{array}$ & $\begin{array}{l}+++ \\
++\end{array}$ \\
\hline $\begin{array}{l}+++ \\
+++ \\
+++ \\
+++ \\
+++ \\
+++ \\
+++\end{array}$ & $\begin{array}{l}+++ \\
+++ \\
+++ \\
+++ \\
+++ \\
+++\end{array}$ & $\begin{array}{l}++ \\
+ \\
+++ \\
+ \\
+\end{array}$ & $\begin{array}{l}+ \\
+\end{array}$ & $\begin{array}{l}+++ \\
+++ \\
+++ \\
+++ \\
+++ \\
++ \\
+++\end{array}$ & $\begin{array}{l}+ \\
+ \\
+ \\
+ \\
+ \\
+\end{array}$ & & & $\begin{array}{l}+ \\
+\end{array}$ & $\begin{array}{l}+ \\
+\end{array}$ & $\begin{array}{l}+ \\
+\end{array}$ & + & & $\begin{array}{l}+ \\
+ \\
+\end{array}$ & $\begin{array}{l}+ \\
+\end{array}$ \\
\hline $\begin{array}{l}+++ \\
+++ \\
+++ \\
+++ \\
+++ \\
+++ \\
+++ \\
+++ \\
++ \\
++ \\
+++ \\
+++ \\
+++ \\
+++ \\
++ \\
+++ \\
+++ \\
+++ \\
+++ \\
+++ \\
++ \\
+++ \\
++ \\
++ \\
+++ \\
+++\end{array}$ & $\begin{array}{l}+++ \\
+++ \\
+++ \\
+++ \\
+++ \\
+++ \\
\\
+++ \\
+ \\
++ \\
+++ \\
+++ \\
+++ \\
+++ \\
+++ \\
+++ \\
+++ \\
+++\end{array}$ & $\begin{array}{l}+ \\
+ \\
++ \\
+ \\
\\
++ \\
+ \\
+++ \\
+ \\
+ \\
+ \\
+ \\
+ \\
+ \\
+ \\
+ \\
+ \\
+\end{array}$ & $\begin{array}{l}+ \\
+\end{array}$ & $\begin{array}{l}+++ \\
++ \\
++ \\
+++ \\
+ \\
+++ \\
++ \\
+++ \\
+++ \\
+++ \\
+++ \\
++ \\
+++ \\
+++ \\
++ \\
+++ \\
+ \\
++\end{array}$ & $\begin{array}{l}+ \\
++ \\
+ \\
+ \\
+ \\
+ \\
+ \\
+\end{array}$ & + & $\begin{array}{l}+ \\
++\end{array}$ & $\begin{array}{l}+++ \\
+++ \\
++ \\
++ \\
+++ \\
+ \\
+ \\
+++\end{array}$ & + & & & +++ & $\begin{array}{l}+ \\
+\end{array}$ & $\begin{array}{l}+++ \\
++ \\
+ \\
+++\end{array}$ \\
\hline
\end{tabular}

bedeuten Flecke von nicht identifizierten Substanzen. 
in nur relativ geringen Tiefen gefangen. Der Histidin-Gehalt war bei den meisten Knochenfischen der kalten und tieferen Gewässer des Nordatlantiks gering.

Arginin lag bei den schnellen Schwimmern nur in geringer Menge vor oder war nicht nachweisbar. Die höchsten Konzentrationen traten bei der Seezunge auf.

Hoher Gehalt an B-Alanin wurde mit Ausnahme des Meeraals nur bei Fischen aus kaltem Wasser des Raumes Island-Grönland beobachtet.

Die Prolinmengen schwankten nicht nur stark von Art zu Art, sondern auch bei den Individuen derselben Art (vgl. Schaefer, 1959). Mit Ausnahme des Meeraals lagen größere Mengen von Prolin nur bei den im Sommer gefangenen Fischen vor.

Starke Konzentrationen von Oxyprolin wurden vorwiegend bei den im Sommer im nördlichen Raum gefangenen Fischen beobachtet. Die aktivsten Schwimmer besaßen nur wenig Oxyprolin.

Anserin wurde bei allen Gadiden gefunden, die Befunde von Shewan (1953) und Ranke (,g.b.F.“, 1959) werden damit bestätigt. In der Ordnung Perciformes wurde Anserin nicht beobachtet bei den Percoidei mit Ausnahme von Seebarsch, Blennioidei, Scombroidei, Cottoidei mit Ausnahme von den Knurrhahn-Arten, und Callionymoidei. Von den Plattfischen scheinen nur die Bothidae und Heilbutt und Doggerscharbe Anserin zu haben, bei ihnen kam auch "10" vor. Bei den übrigen Plattfischen wurde Anserin nicht gefunden, es lag vermutlich in zu geringer Menge vor.

Während der ersten Fahrt wurden annähernd gleichgroße Streifenbrassen aus demselben Netzfang aus $72 \mathrm{~m}$ Tiefe in gutdurchlüftete Bordaquarien der Größe $100 \times 49,3 \times 49 \mathrm{~cm}$ gesetzt. Von je drei Tieren wurden sofort nach dem Fang sowie nach 5-, 24-, 48-, 51-, 74-, 96- und 120stündiger Hälterung Muskelproben entnommen und aufgearbeitet. Auf den Papierchromatogrammen war ein deutlicher Unterschied in der Größe und Intensität des Lysinflecks festzustellen. Die Lysinmenge war sofort nach dem Fang schwach, ab 6stündiger Hälterung stark. Der Unterschied war auf zahlreichen, wiederholt angefertigten Chromatogrammen deutlich sichtbar, Analysenfehler sind also ausgeschlossen. Ferner wurde ein Unterschied in den Mengen von Leucin/ Isoleucin beobachtet. Die Papierchromatogramme der 6 Stunden lang. gehälterten Tiere zeigten nur schwache Konzentrationen beider Aminosäuren an.

\section{Diskussion}

Die Ergebnisse der quantitativen Analysen von Gesamt- und Aminostickstoff decken sich größenordnungsmäßig mit den von RANKE (1959) ermittelten Konzentrationen, wenn auch die oberen und unteren Grenzen auf Grund des erweiterten Artenmaterials, das auch aus verschiedenen Meeren stammt, verschoben sind.

Es bedarf natürlich sehr eingehender und langwieriger Untersuchungen, um festzustellen, welche Rolle den einzelnen freien Aminosäuren zukommt. Wenn auch mit den vorliegenden Untersuchungen weiteres Analysenmaterial gebracht wird, so konnten doch diese biologisch sehr interessierenden Probleme nicht gelöst werden. Es soll aber im folgenden versucht werden, die Fülle der möglichen Funktionen einzuengen. Anhand der bereits veröffentlichten 
und der jetzt vorgetragenen Befunde soll festgestellt werden, ob bestimmte freie Aminosäuren in bestimmten Größenordnungen für eine bestimmte Fischgruppe charakteristisch sind, wenn $j a$, in welcher Beziehung eine Gemeinsamkeit bei diesen Arten vorliegt, oder inwieweit sich diese Arten von anderen unterscheiden. Handelt es sich um Aminosäuren, deren Konzentrationen innerhalb derselben Fischart stark schwanken, dann soll erkundet werden, unter welchen Bedingungen die geringste oder die größte Menge auftritt. In vielen Fällen ist das Tatsachenmaterial noch zu gering, sehr häufig werden auch unsere bisherigen zur Diskussion erforderlichen Kenntnisse der Lebensweise und der Umweltfaktoren versagen, handelt es sich doch zum Teil um Arten, von denen eingehende biologische Bearbeitungen fehlen. In einigen Fällen wird es aber doch möglich sein, eine Beziehung einer gegebenen Substanz zu exogenen oder endogenen Faktoren festzustellen. Da die Zahl der untersuchten Arten bereits beträchtlich ist, soll auch versucht werden festzustellen, ob das Grundmuster der freien Aminosäuren verwandtschaftliche Beziehungen zwischen den einzelnen Arten erkennen läßt.

Es wird ausdrücklich darauf hingewiesen, daß nur sehr markante Unterschiede der aus den Papierchromatogrammen ersichtlichen Mengen diskutiert werden.

Frühere Untersuchungen hatten ergeben, daß das Grundmuster der freien Aminosäuren bei verschieden alten Individuen derselben Art (Rotbarsch, Kabeljau, Schellfisch), die aus demselben Fang stammten und sofort nach dem Fang analysiert wurden, papierchromatographisch einheitlich ist. Geringere Abweichungen waren nur bei Prolin, Leucin/Isoleucin und Histidin zu konstatieren. Starke Unterschiede in diesem Muster ergaben sich aber, wenn Fische derselben Art von verschiedenen Fangplätzen stammten bzw. zu verschiedenen Jahreszeiten untersucht wurden (Schatfer, 1961 a).

Das Vorkommen sehr großer Mengen Histidin bei Hering, Makrele und anderen pelagischen Fischen wurde schon von mehreren Autoren beobachtet (Amano und Bito, 1953; Shewan, 1955; Lukton und Olcott, 1958; Hughes, 1959 ; u. a.). Der Befund kann in Zusammenhang mit dem starken Hämoglobingehalt derselben Fische (Schaefer, 1961 b) gebracht werden: Je aktiver die Fische sind, um so höher ist ihre Blutfarbstoffkonzentration (vgl. Gray, 1954). Histidin spielt bekanntlich eine große Rolle bei der Hämoglobinbildung. Starken Histidingehalt weisen auch weitere pelagische oder relativ agile Arten auf, welche weniger Hämoglobingehalt besitzen. Umgekehrt ist die bei einem der aktivsten Schwimmer des Nordens, dem schwarzen Heilbutt, gefundene Menge Histidin gering, bei dem trägen Wolfsfisch mit wenig Blutfarbstoff aber recht hoch. Auch bei dem trägen Rotbarsch wurde zuweilen, standortabhängig, beträchtliches Histidinvorkommen beobachtet. Ein Zusammenhang mit dem Fettgehalt scheint nicht zu bestehen, da sich unter den histidinreichen Fischen auch etliche Magerfische befinden. Eine Abhängigkeit vom Standort ist insofern gegeben, als die hier untersuchten pelagischen Fische in geringen Tiefen gemäßigter Meere vorkamen. Die Muskulatur der meisten bei Island-Grönland gefangenen Arten enthielt entsprechend wenig Histidin.

Sehr agile Fische enthielten nur wenig oder kein nachweisbares Arginin und Oxyprolin, aber relativ viel Valin, Leucin und Isoleucin. Fleck „10" war bei ihnen nicht erkennbar. Bei anderen Fischen mit hohem Histidingehalt kam Arginin aber in höheren, Leucin, Isoleucin und Valin in geringeren Mengen 
vor. Nach den bisherigen Untersuchungen ist die Gruppe der aktiven Fische also durch das gleichzeitige Vorkommen von sehr starken Mengen Histidin, stärkeren Mengen Valin, Leucin und Isoleucin und sehr geringen Mengen oder durch Fehlen von Arginin, Oxyprolin und „10“ von den übrigen Fischarten abzutrennen, der Pool der freien Aminosäuren hat also hier ein recht charakteristisches Muster.

Ein weiterer auffälliger Befund ist das unterschiedliche Vorkommen bestimmter Substanzen vorwiegend bei Fischen aus bestimmten Gebieten. GröBere Mengen von Gesamtstickstoff kamen vornehmlich bei Fischen aus dem Raum Island-Grönland vor, alle als „arktisch" einzuordnenden Arten besaßen einen sehr hohen Aminostickstoff-Gehalt. Ferner wurden B-Alanin und Oxyprolin in hohen Konzentrationen fast nur bei einer Reihe von Arten aus den nördlichen Meeren gefunden, während umgekehrt die Fische mit weniger ß-Alanin und Oxyprolin und größeren Mengen von Substanz "1" fast alle aus Nordsee und Kanal stammten. In der Nordsee wurde B-Alanin in größerer Konzentration nur beim Meeraal beobachtet, nach RANke auch beim Aal. Diese Aminosäuren sind aber nicht allgemein charakteristisch für alle borealen und arktischen Formen, da manche dieser Arten auch geringere Konzentrationen aufwiesen. Daß die Doggerscharbe der Nordsee niedrige Mengen $ß$-Alanin aufwies, kann mit dem Obengesagten in Zusammenhang stehen. Auf Station 2526 wurde nur wenig, auf 2620 ein sehr starker Gehalt beobachtet. Hierbei ergab sich die Feststellung, daß alle auf 2620 gefangenen Knochenfische mittlere bis starke Mengen B-Alanin enthielten, auf 2526 aber weder die Doggerscharbe noch der schwarze Heilbutt. Weitere Unterschiede im Aminosäuremuster der Doggerscharbe auf diesen beiden Stationen betrafen das mengenmäßige Auftreten von Histidin, Oxyprolin und Fleck „5“. Die Ursachen dieser Verschiedenheit sind noch nicht bekannt, möglicherweise spielt hier die Nahrung eine Rolle, da beide Stationen biologisch verschieden sein dürften. Möglicherweise gehören die Doggerscharben der Nordsee und die von Station 2526 der Unterart Hippoglossoides platessoides limandoides, die von 2620 der Unterart H. platessoides platessoides (nach Norman, 1934) an. Dann könnten die Unterschiede also genetisch bedingt sein. Die Möglichkeit genetischer Fixierung bedarf aber noch weiterer Untersuchungen.

$\mathrm{Daß}$ Oxyprolin und $\beta$-Alanin bei Fischen aus der Nordsee in vorwiegend geringer Konzentration gefunden wurde, geht auch aus den Arbeiten von Shewan, Ranke und Jones hervor.

Über die Bedeutung dieser beiden Aminosäuren ist wenig bekannt. Abgesehen von seiner Entstehung aus anderen Aminosäuren, kommt B-Alanin als Bestandteil der Peptide Carnosin und Anserin und der Pantothensäure vor, doch wurden diese Peptide nicht bei allen Fischen mit hohem Gehalt an ß-Alanin nachgewiesen. Es ist auch keine Beziehung zu Histidin erkennbar. Oxyprolin ist ein wichtiger Bestandteil der Skleroproteine. Collagen kommt in sehr beträchtlicher Menge in der Fischhaut, in den Schuppen und im Bindegewebe vor. Nach Harkness (1961) ist die Kontraktionstemperatur des Fischcollagens mit der Außentemperatur korreliert, sie ist am geringsten bei Kaltwasserfischen. Da der Oxyprolingehalt des Collagens umgekehrt proportional der Kontraktionstemperatur ist, wäre das Vorhandensein größerer Mengen von Oxyprolin bei nordischen Fischen verständlich. Da Oxyprolin in großer Konzentration aber nicht bei allen nordischen Fischen auftritt, muß 
ein mengenmäßig sehr unterschiedliches Vorkommen von Art zu Art angenommen werden.

$\mathrm{Zu}$ den Befunden, daß sich manche Fischarten aus nördlichen Gewässern durch mengenmäßig unterschiedliches Vorhandensein von bestimmten Substanzen von denen der gemäßigten Zonen unterscheiden, gibt es weitere Parallelen. Die nördlichen Arten besitzen mehr Trimethylaminoxyd als die Fische der Nordsee (ShEwan, 1951), haben vorwiegend geringeren Hämoglobingehalt und weisen niedrigere Erythrozytenzahlen auf (NAUmov, 1956; SchaEfer, $1961 \mathrm{~b}$ ).

Die Ursachen der Abhängigkeit der Mengen einiger freier Aminosäuren vom Standort sind noch nicht klar. Wenn auch in unserem Fall keine nennenswerte Schwankungen im Salz- (Ausnahme Station 2620) und Sauerstoffgehalt auf den Fischgründen zu bemerken waren, so sind doch manche Stationen, selbst so benachbarte wie 2523 und 2526, hydrographisch (DiETrich et al. 1961) und biologisch, z. B. Planktonvorkommen (GILlBRICHT, persönliche Mitteilung), unterschiedliche Nährfauna u. a. verschieden. Daß solche standortabhängige Faktoren wie Ernährung, Temperatur, Salzgehalt auf den Bestand der freien Aminosäuren in der Fischmuskulatur einwirken können, wurde schon wiederholt nachgewiesen. So variieren die Konzentrationen einiger Aminosäuren bei verschiedenartiger Ernährung (FARRIs, 1958; LukTon, 1958). Eine Beeinflussung des Aminosäurepools durch unterschiedliche Salzgehalte wurde von Ranke (1959) und Allen (1961) beobachtet. Florkin (1956) setzte Wollhandkrabben in Aquarien von $10^{\circ}$ und $1-3^{\circ}$. Bei den abgekühlten Tieren trat Erniedrigung der Prolinmengen auf. Die Befunde zeigen die Bedeutung der freien Aminosäuren für die Osmoregulation.

Bei anderen Stoffen finden wir ebenfalls ähnliche standortbedingte $\mathrm{Ab}$ hängigkeiten, z. B. durch verschiedenes Nahrungsvorkommen oder durch Nahrungskonkurrenz. Nach Morawa (1958) treffen die Maränen im stark eutrophierten Plöner See bedeutend bessere Nahrbedingungen an und weisen daher höheren Fettgehalt auf als die des Selenter Sees, wo hohe Individuenzahlen auf niedrige Planktonmengen stoßen. Der Fettgehalt variiert beim Hering von Fangplatz zu Fangplatz (SASA, 1958) - hier können auch verschiedene Reifestadien von Einfluß sein -, der Wassergehalt des Kabeljaus hängt ebenso vom Standort ab (Love, 1960). Somit erfahren die eigenen Befunde (Schaefer, 1961 a), daß artgleiche Tiere von verschiedenen Fangplätzen unterschiedliche Mengen bestimmter freier Aminosäuren enthalten, eine gewisse Bestätigung.

Eine Beziehung des Auftretens von Oxyprolin zur Wassertemperatur und Fangtiefe ist nicht ersichtlich. Die B-Alanin-reichen Fische aber stammten alle mit Ausnahme des Meeraals aus relativ kaltem Wasser. Fische mit niedrigem Gesamtstickstoff wurden in vorwiegend geringen Tiefen gefangen. Bei den übrigen Aminosäuren ergibt sich aber kein bemerkenswerter Zusammenhang mit diesen beiden Faktoren.

Weiterhin sind jahreszeitliche Unterschiede festzustellen. Die Gesamtstickstoff-Mengen lagen im Sommer durchweg höher als im Spätwinter. Bei den im Spätwinter gefangenen Kabeljau und Brosmen wurde kaum Oxyprolin nachgewiesen, während die Konzentrationen im Sommer mittel bis stark waren. Fische mit hohem Prolingehalt (Prolin ist ebenfalls ein Hauptbestandteil des Collagens) stammten mit Ausnahme des Meeraals alle aus Sommerfängen. Prolin war bei zu verschiedenen Jahreszeiten gefangenen Tieren 
im Sommer stärker vorhanden als im Spätwinter (Kabeljau, Bastardmakrele), während die Tiefenbarsche im Winter höhere Mengen an Glutaminsäure und Alanin-Threonin aufwiesen (Schaefer, 1961 a). Der Threoninfleck war bei Bastardmakrelen im Sommer größer.

Nach Macleod (1959) ist der Gehalt an Oxyprolin bzw. Collagen starken jahreszeitlichen Schwankungen unterworfen.

Über jahreszeitliche Schwankungen im Muskelpool der freien Aminosäuren liegen auch von Jones (1955), Hughes (1959) und Ranke (1959) Beobachtungen vor. Diese Unterschiede können zum Teil mit den Laichzeiten in Zusammenhang stehen (JoNEs, 1955; SASA, 1958). Jedoch kann auch hier ein Einfluß von Ernährung, Temperaturänderung des Wassers und von Wanderungen vorliegen. Parallelen dazu finden sich in einer zusammenfassenden Arbeit von TARR (1959).

Eine Beeinflussung des Aminosäurenbestandes ist auch aus dem Hälterungsversuch mit Streifenbrassen erkennbar. Eine große Schwankungsbreite der Lysinmengen bei den untersuchten Fischen ist nicht anzunehmen, da sämtliche im Aquarium gehaltenen Brassen dieselbe Fleckengröße des Lysins auf den Chromatogrammen zeigten. Salz- und Sauerstoffgehalt blieben in den gut mit Meerwasser durchspülten Aquarien unverändert. Wenn auch die direkte Ursache der Mengenveränderungen von Lysin sowie von Leucin/Isoleucin nicht zu erkennen ist - es ist an Druckunterschiede, Temperaturschwankungen, Veränderungen der Osmoregulation durch Hautverletzungen u. a. zu denken - so geht doch aus dem Versuch hervor, daß die Analyse von in Aquarien gehälterten Seefischen nicht in jedem Fall mit der von frischgefangenen Fischen übereinstimmt. Das wurde durch Untersuchungen des Blutes derselben Streifenbrassen bestätigt (Schaefer, 1961 b). Eine Veränderung des Aminosäurenbestandes in der Fischmuskulatur innerhalb weniger Stunden ist durchaus möglich (RANKE, 1959).

Verschiedentlich ist in den biologischen Disziplinen der Versuch gemacht worden, biochemische Befunde in die Systematik einzuordnen, um auf diese Weise Verwandtschaftsbeziehungen zu bestätigen oder um strittige Fragen zu überprüfen. Shewan (1953) und Ranke (1959) fanden, daß Anserin bzw. der Fleck „g. b. F." (RANKE) u. a. bei allen Gadiden anzutreffen ist und somit als systematisches Merkmal verwertbar ist. Weitere „Familienmerkmale" wurden von den Autoren beschrieben. Die vorliegenden Untersuchungen konnten das Auftreten von Anserin bei weiteren Gadiden bestätigen. Aber nur bei einem Teil der Plattfische gelang es, Anserin nachzuweisen. Da mit Ausnahme der Scheef snut Anserin bei den positiven Plattfischen nur schwach vorhanden war, bleibt die Möglichkeit offen, daß auch die Fische mit negativem Befund das Peptid in unter der Nachweisbarkeitsgrenze liegenden Konzentrationen besitzen. Dasselbe gilt für Knurrhahn von Station 2477. Die übrigen Funde von Anserin in anderen Arten (Ranke, Shewan, Yudaev, 1950) wurden durch die eigenen Untersuchungen, soweit sie diese Arten einbezogen, bestätigt. Demnach tritt Anserin u. a. bei den bisher untersuchten Arten der Plattfische, Gadiformes und Anguilliformes auf. In der Ordnung der Perciformes werden aber Unterschiede beobachtet: bei einem Großteil der Unterordnungen und Familien war Anserin in Übereinstimmung mit RANKE nicht nachzuweisen, wohl aber bei der Brachsenmakrele (Shewan, 1955), dem Seebarsch und zum Teil bei den Cottoidei: positiv: Seeskorpion (RANKE) und Knurrhahn; negativ: Kleiner Scheibenbauch (RANKE), Rotbarsch, Seehase und Cottunculus. Ähn- 
liches gilt für die Clupeiformes: Finte (Shewan, 1955) und Hering negativ, zwei Salmo-Arten aus dem Süßwasser (Ranke; Carnosin?) und Glasauge positiv. Es erscheint daher der Schluß berechtigt, daß es noch zweifelhaft ist, das Vorkommen von Anserin unbedingt als systematisches Merkmal anzusehen. Da Anserin wie auch Carnosin eine wichtige Rolle im Stoffwechsel spielen (Severin, 1958), ist ihr Auftreten vielleicht auch mit noch nicht erkennbaren physiologischen Faktoren zu korrelieren. SHEwan (1955) weist darauf hin, daß die Brachsenmakrele (Brama raii) (Anserin positiv) weniger pelagisch ist als die Makrele und der Hering (Anserin negativ) und so eine Mittelstellung zwischen diesen aktiven Fischen und den Gadiden (Anserin positiv) einnimmt. Jedoch besteht kein Zusammenhang mit dem Vorkommen von $ß$-Alanin und Histidin bzw. Methylhistidin. Da nicht bei allen Arten nachgeprüft wurde, ob es sich um Anserin oder Carnosin handelt, bleibt die Frage nach dem Vorkommen von Carnosin offen. Nach Lukton und Oцcotт (1958) und Yudaev (1950) kommt Carnosin stärker bei Süßwasserfischen vor.

Beziehungen anderer freier Aminosäuren zu den genannten ökologischen und physiologischen Faktoren sind nicht erkennbar. Möglicherweise liegt ihre Bedeutung in der Hauptsache in ihrer Funktion als Eiweißbausteine. Dagegen sprechen nicht die Befunde von Jones (1954), daß einige dieser Substanzen im Verlaufe des Jahreszyklus Konzentrationsunterschiede aufweisen, da Reifung und Laichzeiten von großem Einfluß auf den Eiweißbestand sind. Hingegen scheint die Bedeutung einiger der ausführlicher diskutierten Aminosäuren zum Teil eine doppelte zu sein: sie sind erstens (mit Ausnahme des ß-Alanins) Eiweißbausteine und als solche Konzentrationsschwankungen durch Auf- und Umbau unterworfen und treten in den Zwischenphasen im Pool der freien Substanzen auf, zweitens aber dürften sie, wie auch manche Nichteiweiß-Aminosäuren, bei anderen physiologischen Vorgängen eine Rolle spielen, die wir im Einzelnen noch nicht kennen.

Vergleicht man die Muster der freien Aminosäuren der verschiedensten Arten miteinander, dann findet man eine erstaunliche Vielgestaltigkeit, die den Eindruck erweckt, als besäße jede Art in der Muskulatur einen eigenen spezifischen Aminosäurepool. Nach Florkin (1956) soll jede Art außer der spezifischen Zusammensetzung noch eine spezifische Durchschnittskonzentration dieses Pools besitzen, die sich besonders beim Vergleich von Arten aus verschiedenen Tierklassen bemerkbar macht. Ranke sieht „die Artspezifität der freien Aminosäurenbestände im Vorhandensein unterschiedlicher Mengen der allen Tieren zukommenden freien Aminosäuren". Im allgemeinen wird diese Aussage auch durch die vorliegenden Untersuchungen bestätigt, da bei den einzelnen Arten einmal die eine, einmal die andere Aminosäure in stärkerer oder schwächerer Konzentration vorkommt. Dies sieht nach genetischer Fixierung des Grundmusters der freien Aminosäuren aus. Jedoch lassen die Beobachtungen, daß die Aminosäurengarnituren durch die Einflüsse von Umweltsund inneren Faktoren (z. B. Reifung) sehr starken Veränderungen ausgesetzt sind, die Artspezifität des Aminosäurenmusters zweifelhaft erscheinen, was auch aus der Arbeit von FarRis (1958) ersichtlich ist. Selbst Florkin und RANKE weisen darauf hin, daß die Konzentrationen einiger freier Aminosäuren durch den Einfluß von Salzgehalt- und Temperaturänderungen sehr stark schwankten, woraus der Schluß auf die Bedeutung von vorzugsweise Glycin und Prolin für die Osmoregulation gezogen wurde. Es ist daher denkbar, daß 
unter bestimmten Bedingungen einige Arten auch mengenmäßig den gleichen Bestand an freien Aminosäuren haben könnten. Nach den bisherigen Befunden dürfte es so sein, daß man verschiedene Arten auf Grund des Musters der freien Aminosäuren unterscheiden kann, wenn sie aus demselben Fang stammen, wenn also wenigstens die Umweltbedingungen mehr oder weniger identisch sind. Ranke konnte daher schon eher von einer Artspezifität des Musters sprechen, da die von ihm untersuchten Seefische alle aus demselben Habitat, wenngleich auch aus verschiedenen Jahreszeiten, stammten.

Im Vorstehenden wurde der Versuch gemacht, verschiedene auffällige Befunde in Zusammenhang mit verschiedenen Faktoren zu bringen. Dies kann aber auch nur als ein Versuch angesehen werden, da die Größe des Materials zu definitiven Aussagen nicht ausreicht. Angesichts der normalerweise vorhandenen Streuungen der Konzentrationen von Körperbausteinen und Metaboliten sind exakte Gesetzmäßigkeiten auch nur bei einer Fülle von Proben aufzufinden. Zudem ist zu erwarten, daß in vielen Fällen verschiedene Faktoren zusammenwirken. So kann das stärkere Vorkommen gewisser Substanzen vornehmlich in bestimmten Gebieten auch mit der Fangtiefe und der dort herrschenden Wassertemperatur oder das mengenmäßig unterschiedliche Auftreten bestimmter Aminosäuren bei artgleichen Fischen von verschiedenen Fangplätzen auch mit verschiedenartiger Ernährung oder anderen Faktoren kombiniert sein. Möglicherweise variiert die Art der Zusammensetzung der Nahrungstiere ebenfalls von Ort zu Ort. Nicht zu verkennen ist der Einfluß des gerade vorliegenden Reifestadiums. Die Analyse der im natürlichen Lebensraum gefangenen Tiere vermag nicht allein zu entscheiden, durch welche spezielle Faktoren die Mengenverhältnisse der Substanzen beeinflußt werden. So sind die Bezeichnungen „standort- oder jahreszeitlich bedingte Faktoren oder Abhängigkeiten" eigentlich nur Überbegriffe, die wiederum miteinander zusammenhängen können, für eine Mehrzahl von Faktoren. Hier könnte das Experiment mit isolierten Fischen Auskunft darüber geben, inwieweit der einzelne der möglichen Faktoren eine Bedeutung für die chemische Zusammensetzung mancher Organe haben kann. Dabei darf aber nicht außer acht gelassen werden, daß diese Zusammensetzung auch auf der gleichzeitigen, in verschiedener Weise gerichteten Einwirkung mehrerer Faktoren beruhen kann. Es ist daher zweifelhaft, ob diese Einzelfragen exakt beantwortet werden können, da es sich um sehr komplexe Geschehen handeln wird. Somit dürfte vorerst, zumindest für die Fragestellung dieser Arbeit, die Anwendung der genannten Überbegriffe genügen, weil ja lediglich gezeigt werden sollte, daß die chemische Zusammensetzung der Fischmuskulatur nicht in allen Fällen artspezifisch ist, sondern mancherlei Einflüssen unterliegen kann.

Unsere Kenntnisse gerade von den nordischen Fischen hinsichtlich Aktivität, Ernährung, Laichzeiten, Collagen- und Vitamingehalt usw. sind zum Teil noch recht dürftig, da viele dieser Arten keine große wirtschaftliche Bedeutung haben. Wenn dennoch eine Ordnung der Befunde gewagt wurde, so war nicht nur die Absicht maßgebend, eine vergleichende Zusammenstellung der Analysenergebnisse bei verschiedenen Arten zu geben, sondern auch eine Anregung zu weiteren Untersuchungen über das Vorkommen und vor allem über die Funktion bestimmter freier Aminosäuren und ähnlicher Substanzen zu vermitteln, da diese Verbindungen bzw. ihre Abbauprodukte nicht nur eine große Rolle im Stoffwechsel spielen, sondern auch als Geruchs- und Geschmacksstoffe (BRAMSTEDT, 1957) sowie als Indikatoren der Verderbnis des 
Fischfleisches, wie zahlreiche Autoren gezeigt haben, von überragender Bedeutung ist.

\section{Zusammenfassung}

Das Vorkommen von freien Aminosäuren und verwandten Verbindungen in alkoholischen Extrakten der Muskulatur von 38 Fischarten aus verschiedenen Meeren wurde papierchromatographisch untersucht. Die Arten unterscheiden sich qualitativ nur durch das Vorkommen von noch nicht identifizierten Verbindungen, in quantitativer Hinsicht jedoch durch mengenmäßig unterschiedliches Auftreten einzelner freier Aminosäuren.

Es wurde versucht, die Befunde biologisch einzuordnen.

Die sehr aktiven Fische unterscheiden sich von den übrigen Arten durch sehr starkes Vorkommen von Histidin und durch stärkere Konzentration von Leucin, Isoleucin und Valin und durch geringe Mengen von Arginin, Oxyprolin und Substanz „10“.

$\beta$-Alanin und Oxyprolin werden in starken Konzentrationen vornehmlich bei Arten aus dem Bereich Island-Grönland gefunden. Fast alle $\beta$-alaninreichen Fische stammen aus kalten Gewässern. Fische mit niedrigem Gehalt an Gesamtstickstoff wurden in vorwiegend geringen Wassertiefen gefangen, während die arktischen Arten sehr hohen Aminostickstoffgehalt haben.

Die Gesamtstickstoff-Mengen liegen im Sommer höher als im Spätwinter. Prolin und Oxyprolin kommen in großen Mengen vornehmlich bei im Sommer gefangenen Fischen vor.

Ein Versuch mit Streifenbrassen zeigte, daß durch das Einsetzen und Halten in Bordaquarien Mengenveränderungen einiger freier Aminosäuren auftreten.

Die Einflüsse von Standortbedingungen, Ernährung und Jahreszeiten auf den Gehalt an freien Aminosäuren und anderen Substanzgruppen, die systematische Bedeutung von Anserin und das Problem der Artspezifität des Musters der freien Aminosäuren werden diskutiert.

\section{Summary}

Alcoholic extracts of the skeletal muscle of 38 freshly caught marine fish species from different seas were analysed for free amino acids and related compounds by means of paper chromatography. The species differ qualitatively by the occurrence of unidentified substances, quantitatively by different concentrations of some free amino acids.

It was tried to bring the results in a biological order.

The active fishes differ from the other species by the occurrence of very large concentrations of histidine, of relatively large amounts of leucine, isoleucine, and valine and of very little quantities of arginine, hydroxyproline and the unidentified substance "10".

Large concentrations of $\beta$-alanine and hydroxyproline were found mainly in fishes from Iceland-Greenland. Nearly all the fishes rich of $\beta$-alanine were caught in cold waters. Fishes with small amounts of total nitrogen have been caught mainly in small depths whilst the arctic species contained important concentrations of amino nitrogen. 
The concentrations of total nitrogen were higher in summer catches than in winter ones. Proline and hydroxyproline were found in large amounts mainly in fishes caught in summer months.

Experiments with Spondyliosoma cantharus kept in well aereated sea water tanks aboard the ship showed variations in the concentrations of free lysine and leucine/isoleucine.

Possible influences of fishing place conditions, food and seasons on the concentrations of free amino acids and other substances, the systematical significance of anserine and the problem of the species specifity of the pattern of free amino acids were discussed.

Für die Anregung der Arbeit und freundliche Unterstützung danke ich Herrn Prof. Dr. Bramstedt, Hamburg. Die Vorarbeiten konnten Dank der gütigen Erlaubnis von Herrn Prof Dr. KüHNAU im Physiologisch-Chemischen Institut, Hamburg, erfolgen. Die Arbeiten wurden in der Fischereibiologischen Abteilung des Instituts für Meereskunde, Kiel, und in der Biologischen Anstalt Helgoland durchgeführt. Den Herren Institutsdirektoren danke ich herzlich für die Überlassung eines Arbeitsplatzes. Besonderer Dank gebührt den Herren Prof. Dr. Bückmann, Prof. Dr. Kändler und Dr. Aurich für vielfache Unterstützung.

\section{Literaturverzeichnis}

A ll e n, K., 1961: The effect of salinity on the amino acid concentration in Rangia cuneata (Pelecypoda). Biol. Bull, 121, 419-424.

A $\mathrm{m}$ a $\mathrm{no}, \mathrm{K}$. and B it o, M., 1953: Consequence of free amino acids generated from decomposing fish muscle. Bull. Tokai Reg. Fish. Res. Lab. 6, 83.

A wa para, J., 1948: Application of paper chromatography to the estimation of free amino acids in tissues. Arch. Biochem. 9, 172

B öhnecke, G. und B ü ckmann, A., 1959: Die Expeditionen von FFS „Anton Dohrn“ und VFS "Gauss" im Internationalen Geophysikalischen Jahr 1957/58. Dtsch. Hydrogr. Z., Erg. Hft., Reihe B, Nr. 3, 12-13.

B r a ms ted t, F., 1957: Geschmacks- und Geruchsstoffe im Fischfleisch. Arch. f. Fischereiwiss., 8, 94-103.

D i et rich, G., 1960: Temperatur-, Salzgehalts- und Sauerstoff-Verteilung auf den Schnitten von FFS "Anton Dohrn" und VFS "Gauss" im Internationalen Geophysikalischen Jahr 1957/58. Dtsch. Hydrogr. Z., Erg. Hft., Reihe B, Nr. 4, 1-103.

F a r ris, D. A., 1958: Diet-induced variation in the free amino acid complex of Sardinops coerulea. J. Cons. int. Explor. Mer, 23, 235-244.

Florkin, M., 1956: Vergleichende Betrachtung des stationären Zustandes der nichteiweißgebundenen Aminosäuren der Tiere. 6. Colloq. Ges. Physiol. Chemie, 62-94 Springer-Verlag, Berlin.

Gra y, I. E., 1954: Comparative study of the gill aerea of marine fishes. Biol. Bull. 107, $219-225$.

Hamilton, P. B. and Anders on, R. A., 1959: Ion exchange chromatography of amino acids. Semiautomatic method of operation with cationic exchange resin columns. Anal. Chem. 31, 1504-1512.

H a r kness, R. D., 1961: Biological functions of collagen. Biol. Rev. 36, 399-463.

$\mathrm{Hughes}$, R. B., 1959: Chemical studies on the herring (Clupea harengus). II. The free amino-acids of herring flesh and their behaviour during post-mortem spoilage. J. Sci. Food Agric. 10, 558-564.

J o n e s, N. R., 1954: Factors affecting the free amino acid composition of fresh and iced skeletal muscle of north sea codling (Gadus callarias). Biochem. J. 58, XLVIII.

- 1955: The free amino acids of fish. I. Taurine in the skeletal muscle of codling (Gadus callarias). J. Sci. Food Agric. 6, 4-9.

- 1959: The free amino acids of fish. II. Fresh skeletal muscle from lemon sole (Pleuronectes microcephalus). J. Sci. Food Agric. 10, 282-286.

K lingmüller, V., E rdmann-Mülle r, G. J., R a usch-Stroomann, J.-G. und $\mathrm{Brune}, \mathrm{G} ., 1955$ : Schnellbestimmung wasserdampfflüchtiger Stoffe: Stickstoff nach 
Kjeldahl, Ammoniak, „Acetonkörper“, „flüchtige Fettsäuren“ und Mandelsäure. Arzneim.-Forsch. 5, 105-109.

L o ve, R. M., 1960: Water content of Cod (Gadus callarias L.) Muscle. Nature 185, 692.

L u k t o n, A., 1958: Effect of diet on imidazole compounds and creatine in chinook salmon Oncorhynchus tshawytscha. Nature 182, 1019-1020.

Lukton, A. and Olcott, H. S., 1958: Content of free imidazole compounds in the muscle tissue of aquatic animals. Food Res. 23, 611-618.

M a cLead, R. A., 1959: Some recent contributions to knowledge of the biochemistry of British Columbia fishes. Part II. Changes in the gelatin content of herring with season. Vortrag 3. Ann. Meeting of Fish. Res. Bd. Canada Committee Technol. Inv., Ottawa, Jan. 9.

Morawa, F. W. F., 1958: Einige Beobachtungen über die Schwankungen des Fett- und Wassergehaltes von Fischen aus verschiedenen Umweltverhältnissen. Verh. Internat. Ver. Limnol. 13, 770-775.

N a u mov, W. M., 1956: Ovogenese und Ökologie des Geschlechtscyclus beim MurmanskHering. Knipowitsch Institut Murmansk, IX

Norman, J. R., 1934: A systematic monograph of the flatfishes (Heterosomata). Bd. 1, London, Oxford University Press.

R a n ke, B., 1959: Über die nicht-eiweißgebundenen und eiweißgebundenen Aminosäurenbestände von Fischen, Mollusken und Krebsen. Arch. Fischerciwiss. 10, 117-159.

S a sa, S., 1958: Chemical studies on herring meat. Bull. Fac. Fish. Hokkaido Univ, 8, $319-345$.

S ch a ef er, H., 1959: Uber die Variabilität des Grundmusters freier Aminosäuren in der Rumpfmuskulatur des Rotbarsches, Sebastes marinus L. Dtsch. Hydrogr. Z., Erg. Hft., Reihe B, Nr. 3, 103-107.

- 1961a: On the variation in the pattern of free amino acids in the skeletal muscle of freshly caught oceanic fish from different localities. Rapp. et Proc.-Verb. 149, 167-174.

- 1961b: Methodik und Ergebnisse von Untersuchungen des Erythrozyten- und Hämoglobingehaltes frischgefangener Seefische. Helg. Wiss. Meeresunters. 8, 68-84.

$\mathrm{Severin,S.E.,} \mathrm{and} \mathrm{Yu} \mathrm{Hu}-\mathrm{Yu}$ : The effect of the dipeptides anserine and carnosine on oxydative phosphorylation in isolated mitochondria of the pectoral muscle of the pigeon. Biochimiya (Mosk.) 23, 862-868.

$\mathrm{S}$ hewan, J. M., 1951: The chemistry and metabolism of the nitrogenous extractives in fish. Biochem. Soc. Symposia Cambridge Engl. 6, 28-48

-, Fletcher, L. I., Partridge, S. M., and Brimley, R. C., 1952: Nitrogenous extractives from fresh haddock muscle: fractionation with ion-exchange resins. J. Sci Food Agric. 3, 394-398.

- 1953: The nitrogenous extractives from fresh fish muscle. II. Comparison of several gadoid and elasmobranch species. J. Sci. Food Agric. 4, 565-568.

- 1955: The nitrogenous extractives from fresh fish muscle. III. Comparison of several flat fishes and members of the herring-mackerel group. J. Sci. Food Agric. 6, 99-104.

$\mathrm{S}$ te gemann, H., und $\mathrm{Gr}$ iff in, H. F., 1958: Ein hochempfindliches spezifisches Sprühreagenz zum Nachweis von Oxyprolin. Naturw. 45, 263.

$\mathrm{Sury}$ a $\mathrm{a}$ r a y a n a $\mathrm{R}$ a o, S. V., 1960: Untersuchungen über das Trimethylaminoxyd in Seefischen und Süßwasserfischen. Diss. Mathem.-Naturw. Fak. Hamburg.

Ta r r, H. L. A., 1959: Biochemical changes in fish during maturation. Marine Biology, Proc. 20th Annual Biology Colloquium, Oregon State College, April 1959, 36-50.

Yud a e v, N. A., 1950: Der ß-Alanin-Gehalt im Muskelgewebe der Kaninchen in verschiedenen Stadien der Ontogenese und in den Muskeln einiger Fische. Dokl. Akad. Nauk SSSR, N. S. 71, 717-720.

Zuwerkalow, D., 1956: in Beloserski-Proskurjakow: Praktikum der Biochemie der Pflanzen. Deutscher Verlag der Wissenschaften, Berlin. 